\title{
Signatures of Ultrafast Reversal of Excitonic Order in $\mathrm{Ta}_{2} \mathrm{NiSe}_{5}$
}

\author{
H. Ning, ${ }^{1,2}$ O. Mehio, ${ }^{1,2}$ M. Buchhold, ${ }^{1,2}$ T. Kurumaji® ${ }^{3}$ G. Refael, ${ }^{1,2}$ J. G. Checkelsky, ${ }^{3}$ and D. Hsieh $\oplus^{1,2, *}$ \\ ${ }^{1}$ Institute for Quantum Information and Matter, California Institute of Technology, Pasadena, California 91125, USA \\ ${ }^{2}$ Department of Physics, California Institute of Technology, Pasadena, California 91125, USA \\ ${ }^{3}$ Department of Physics, Massachusetts Institute of Technology, \\ 77 Massachusetts Avenue, Cambridge, Massachusetts 02139, USA
}

(Received 15 June 2020; revised 25 August 2020; accepted 24 November 2020; published 22 December 2020)

\begin{abstract}
In the presence of electron-phonon coupling, an excitonic insulator harbors two degenerate ground states described by an Ising-type order parameter. Starting from a microscopic Hamiltonian, we derive the equations of motion for the Ising order parameter in the phonon coupled excitonic insulator $\mathrm{Ta}_{2} \mathrm{NiSe}_{5}$ and show that it can be controllably reversed on ultrashort timescales using appropriate laser pulse sequences. Using a combination of theory and time-resolved optical reflectivity measurements, we report evidence of such order parameter reversal in $\mathrm{Ta}_{2} \mathrm{NiSe}_{5}$ based on the anomalous behavior of its coherently excited orderparameter-coupled phonons. Our Letter expands the field of ultrafast order parameter control beyond spin and charge ordered materials.
\end{abstract}

DOI: 10.1103/PhysRevLett.125.267602

Exploring new pathways to optically switch Ising-type electronic order parameters is a major theme of current ultrafast science. In recent years, a variety of out-ofequilibrium protocols have been developed for rapidly switching ferromagnetic [1-3], ferrimagnetic [4-6], antiferromagnetic [7-9], and ferroelectric [10-12] order parameters. However, far less is understood about the mechanisms for switching more exotic order parameters that are not of magnetic and charge dipolar type.

A particularly interesting case is the excitonic insulator (EI), a strongly correlated electronic phase realized through condensation of bound electron-hole pairs [13]. The free energy landscapes of the complex electronic order parameter and the real lattice order parameter of an EI are typically characterized by a Mexican hat with continuous U(1) symmetry and a parabola, respectively [Fig. 1(a)]. However, strong electron-phonon coupling (EPC) induces a tilting of both the lattice and electronic potentials [14-18], reducing the $\mathrm{U}(1)$ symmetry to a discrete $Z_{2}$ Ising-type symmetry. Like in magnetic or charge dipole ordered ferroic materials, this leads to two degenerate ground states characterized by order parameters of equal magnitude but opposite phase [Fig. 1(b)].

There is currently no experimental method to switch nor directly measure the phase of an EI order parameter on ultrashort timescales. An alternative strategy is to measure the phase of the coupled structural order parameter. However, existing time-resolved x-ray and electron diffraction techniques are not phase sensitive. Optical phase-resolved second harmonic generation measurements $[12,19,20]$ have been used to measure the phase of structural order parameters in noncentrosymmetric ferroic materials [21-24], but all known EI candidates are centrosymmetric $[25,26]$. The possible presence of $180^{\circ}$ EI domains further complicates such measurements because domain averaging would cause overall signal cancellation.

In this Letter, we demonstrate via theory and experiment a pathway to optically switch an EI order parameter and to probe this reversal through a coherent EI orderparameter-coupled phonon (OPCP). The time evolution
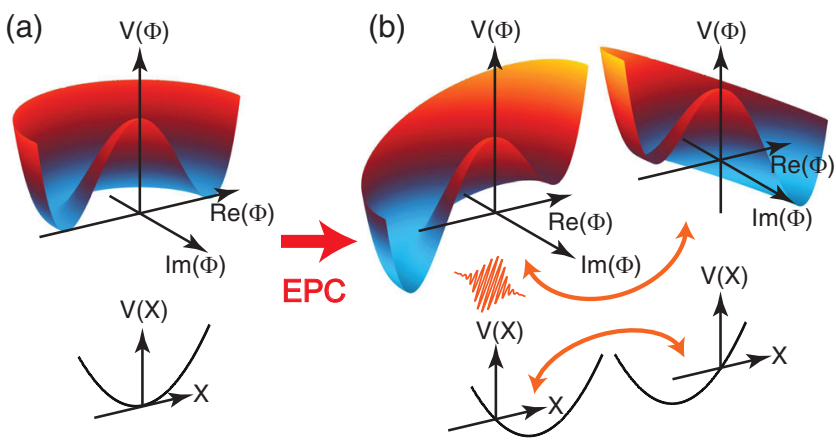

(c)

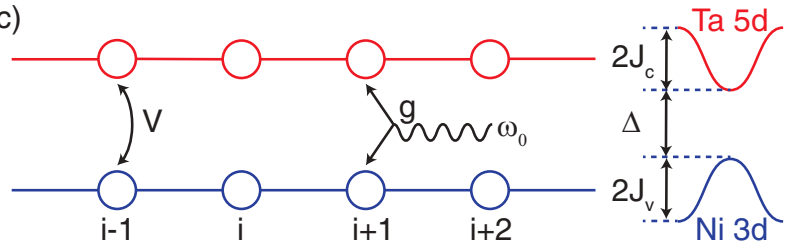

FIG. 1. Schematic of the electronic and structural free energy landscapes (a) without and (b) with EPC. In the latter case, pulsed excitation can drive the system between two degenerate ground states. (c) Schematic of the 1D spinless two-band model. Red (blue) circles on each site $i$ denote Ta $5 d$ conduction band (Ni $3 d$ valence band) states. The microscopic parameters discussed in the main text are defined pictorially. 
of the coupled EI and structural order parameters following impulsive laser excitation are derived from modeling a prototypical system $\mathrm{Ta}_{2} \mathrm{NiSe}_{5}$, which we assume to harbor an EI phase, with an elementary spinless two-band Hamiltonian. Our simulations reveal that the EI order parameter is stably reversed above a critical laser fluence, identifiable indirectly via a saturation of the coherent OPCP amplitude. Using time-resolved coherent phonon spectroscopy measurements, we experimentally verify this scenario and also demonstrate how switching can be controlled through the relative timing between successive laser excitation pulses.

The quasi-one-dimensional (1D) direct band-gap semiconductor $\mathrm{Ta}_{2} \mathrm{NiSe}_{5}$ is reported to undergo an EI transition at a critical temperature $T_{c}=328 \mathrm{~K}[26,27]$, accompanied by a weak orthorhombic-to-monoclinic structural distortion due to EPC [16]. Impulsive laser excitation below $T_{c}$ has been shown to coherently excite at least five distinct Raman-active phonons with frequencies near 1, 2, 3, 3.7, and $4 \mathrm{THz}$. The 1,2, and $4 \mathrm{THz}$ modes are sensitive to the EI transition at $T_{c}[18,28-30]$ and thus constitute the OPCPs, while the 3 and $3.7 \mathrm{THz}$ modes are reportedly not coupled to the EI order parameter and thus serve as a control.

The low energy electronic structure of $\mathrm{Ta}_{2} \mathrm{NiSe}_{5}$ consists of a conduction band with Ta $5 d$ orbital character and a valence band with $\mathrm{Ni} 3 d-\mathrm{Se} 4 p$ hybridized orbital character. The EI instability is well captured by a 1D spinless two-band Hamiltonian with EPC [31-34] [Fig. 1(c)],

$$
\begin{aligned}
H= & \sum_{k}\left(\epsilon_{k} c_{k}^{\dagger} c_{k}+\mu_{k} v_{k}^{\dagger} v_{k}\right)+\sum_{i}\left[V c_{i}^{\dagger} c_{i} v_{i}^{\dagger} v_{i}\right. \\
& \left.+\omega_{0} b_{i}^{\dagger} b_{i}+g\left(b_{i}^{\dagger}+b_{i}\right)\left(c_{i}^{\dagger} v_{i}+v_{i}^{\dagger} c_{i}\right)\right],
\end{aligned}
$$

where $c_{k}^{\dagger}, c_{k}$, and $v_{k}^{\dagger}, v_{k}$ are the fermionic creation and annihilation operators for conduction and valence band electrons with momentum $k$, respectively, and $b_{i}^{\dagger}, b_{i}$ are the bosonic creation and annihilation operators for an OPCP mode of energy $\omega_{0}$ at site $i$. The conduction and valence band dispersions are given by $\epsilon_{k}=(\Delta / 2)+$ $2 J_{c} \sin (k a / 2)^{2}$ and $\mu_{k}=-(\Delta / 2)-2 J_{v} \sin (k a / 2)^{2}$, respectively, where $2 J_{c}$ and $2 J_{v}$ are their bandwidths and $\Delta$ is the band gap [33,34]. An on-site interband electronelectron interaction term $V$ drives the excitonic pairing, and an EPC term $g$ couples the electronic and phononic subsystems. These microscopic parameters have been experimentally determined [33,34].

From Eq. (1), we derive the equations of motion for the EI and structural order parameters, defined as $\Phi_{i}=\left\langle c_{i}^{\dagger} v_{i}\right\rangle$ and $X_{i}=\left\langle b_{i}^{\dagger}+b_{i}\right\rangle$, respectively, in two steps. We first derive the exact expression for the nonequilibrium free energy functional of $\Phi$ and $X$ in the Keldysh path integral framework, where we include the light excitation via Peierls substitution. Then we obtain the equations of motion as the saddle point of the free energy, ignoring population in the conduction bands, spatial fluctuations of the order parameter field, and higher-order contributions $O\left(\Phi^{6}\right)$ (see Supplemental Material [35]),

$$
\begin{aligned}
& i \partial_{t} \Phi=\left[-D(\nabla-i q A)^{2}+m+U|\Phi|^{2}\right] \Phi+2 g^{\prime} X, \\
& \partial_{t}{ }^{2} X=-\left(\omega_{0}^{2}+\frac{2 g^{2} \omega_{0}}{V}\right) X-2 g \omega_{0} \operatorname{Re}(\Phi) .
\end{aligned}
$$

Here $D$ is an effective diffusion coefficient, $q$ is the electron charge, $A$ is the light vector potential, $g^{\prime}$ is the renormalized EPC coefficient for the electronic channel, and $m$ and $U$ are the second- and fourth-order expansion coefficients of the electron-electron interaction term, respectively. These parameters are all functions of $J_{c}, J_{v}, \Delta, g$, and $V$ [35]. We further introduce two phenomenological constants $\gamma_{e}$ and $\gamma_{\mathrm{ph}}$ to account for damping of electronic and structural modes due to thermal fluctuation, whose values can be experimentally determined [35].

We first present a qualitative picture of how order parameter reversal occurs in our model. In equilibrium the electronic potential $V(\Phi)=\frac{1}{2} m \Phi^{2}+\frac{1}{4} U \Phi^{4}+2 g^{\prime} X \Phi$ has either a tilted parabolic $(m>0)$ or tilted Mexican-hat $(m<0)$ form capturing the absence or presence of exciton condensation. For homogeneous optical excitation, one can ignore spatial derivatives of $\Phi$. For pulsed excitation with infrared light, whose frequency well exceeds the electronic Higgs-Goldstone [33,46] and OPCP mode frequencies, one can also average out the fast oscillations of the perturbation and retain only its Gaussian envelope. Under these conditions one can make the simplification $m(t)=-D(\nabla-i q A)^{2}+m \triangleq f(t)+m$, where $f(t)=\alpha F \exp \left[-4 \ln (2) t^{2} / \sigma^{2}\right]$. Here $\sigma$ is the temporal width of the Gaussian pulse, $\alpha$ is a positive constant scaling factor that can be calculated analytically [35], and $F$ is the pump fluence, the only tunable parameter in our model. In the EI phase $(m<0)$, optical excitation therefore acts to instantaneously increase $m(t)$. The subsequent reduction of the EI order parameter, which occurs on a timescale much shorter than $2 \pi / \omega_{0}$, results in a sudden shift in the lattice potential $V(X)=\frac{1}{2} \omega_{0}^{2} X^{2}+$ $2 g \omega_{0} \operatorname{Re}(\Phi) X$ due to EPC [35], launching coherent oscillations through displacive excitation. In the low fluence regime, where the phonon oscillation amplitude is small enough such that $X$ does not change sign, the direction of tilt of both $V(\Phi)$ and $V(X)$ remains unchanged and so no switching occurs. However, above a critical fluence $F_{c}$, where the phonon oscillation amplitude becomes large enough to change the sign of $X$, the tilting of both potentials is reversed and the system can relax into the switched state.

Numerical simulations of our model using experimentally determined material parameters for $\mathrm{Ta}_{2} \mathrm{NiSe}_{5}$ and $\sigma=100 \mathrm{fs}$ were carried out in the EI phase [35]. Figure 2 displays simulation results for $F$ slightly greater than $F_{c}$. 


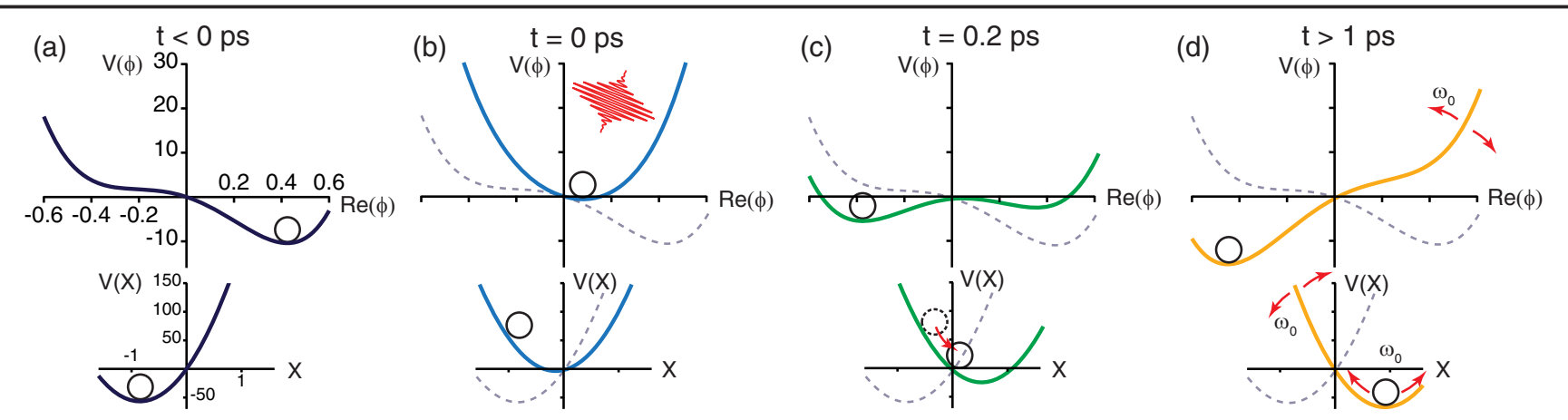

FIG. 2. Simulation results of $V(\Phi)$ and $V(X)$ (defined in main text) for $F>F_{c}$ with experimentally determined parameters [35]. Snapshots of the potential landscapes (solid lines) and the electronic and structural order parameters (circles) are shown (a) in the equilibrium state [reproduced as dashed lines in (b)-(d)], (b) at the moment of excitation, (c) during transit into the reversed state, and (d) in the reversed state before equilibration, where both potentials are modulated at the phonon frequency (red arrows). Axes' scales are the same in all panels.

At the moment of excitation $t=0$ [Fig. 2(b)], there is an instantaneous change in $V(\Phi)$ from Mexican-hat to parabolic form caused by the light-induced enhancement of $m(t)$. The EI order parameter evolves rapidly to the new potential minimum with overdamped dynamics and is quenched within the pulse duration. This leads to a rapid shift in $V(X)$, launching coherent oscillations of the underdamped OPCP, which shakes the electronic potential via EPC at the phonon frequency. Once the pulsed excitation is over, $V(\Phi)$ recovers a Mexican-hat form. However, as $X$ crosses zero within the first half period of oscillation [Fig. 2(c)], the tilting of $V(\Phi)$ is reversed with respect to the prepumped $(t<0)$ case [Fig. 2(a)], sending $\Phi$ toward the new minimum on the negative side. As $\Phi$ crosses zero, the tilt of $V(X)$ is also reversed due to EPC, thus pushing $X$ to the new minimum on the positive side. The system then continues to oscillate about the reversed minima at the phonon frequency until the OPCP is damped out [Fig. 2(d)]. Note that this model treats light as a coherent drive without considering heating- and coolinginduced changes in $m(t)$. However, accounting for the latter merely shifts $F_{c}$ [35].

In lieu of probing the phase of $X$ and $\Phi$, we propose that the reversal can be identified via the pump fluence dependence of the OPCP amplitude. We solve Eqs. (2) and (3) to obtain the time evolution of $\Phi$ and $X$ and then define the OPCP amplitude by its peak height in the fast Fourier transform (FFT) of $X$. A conventional Raman-active phonon is coherently launched through either displacive excitation or impulsive stimulated Raman scattering (ISRS) $[47,48]$ with an amplitude that is linearly proportional to $F$. For $F<F_{c}$, the amplitude of an OPCP also scales linearly with $F$. In this regime, the structural order parameter simply oscillates about the initial potential minimum and thus behaves like a conventional phonon. However, once the initial displacement of $V(X)$ is large enough to enable escape to the opposite minimum $\left(F>F_{c}\right)$, the amplitude ceases to grow. Order parameter reversal is thus marked by a saturation in the amplitude versus fluence curve.

We argue that experimental evidence for this phenomenon already exists in published studies of $\mathrm{Ta}_{2} \mathrm{NiSe}_{5}$. Werdehausen et al. [28] performed ultrafast optical reflectivity measurements at $T=120 \mathrm{~K}$ using a pump photon energy of $1.55 \mathrm{eV}$ and observed clear coherent oscillations of the $1 \mathrm{THz}$ OPCP as well as the uncoupled $3 \mathrm{THz}$ phonon (ISRS). The pump fluence dependence of these two mode amplitudes is reproduced in Fig. 3. The $3 \mathrm{THz}$ mode scales linearly with $F$, consistent with its assignment as a conventional phonon. In contrast, the $1 \mathrm{THz}$ mode scales linearly with $F$ only at low fluences and then saturates above

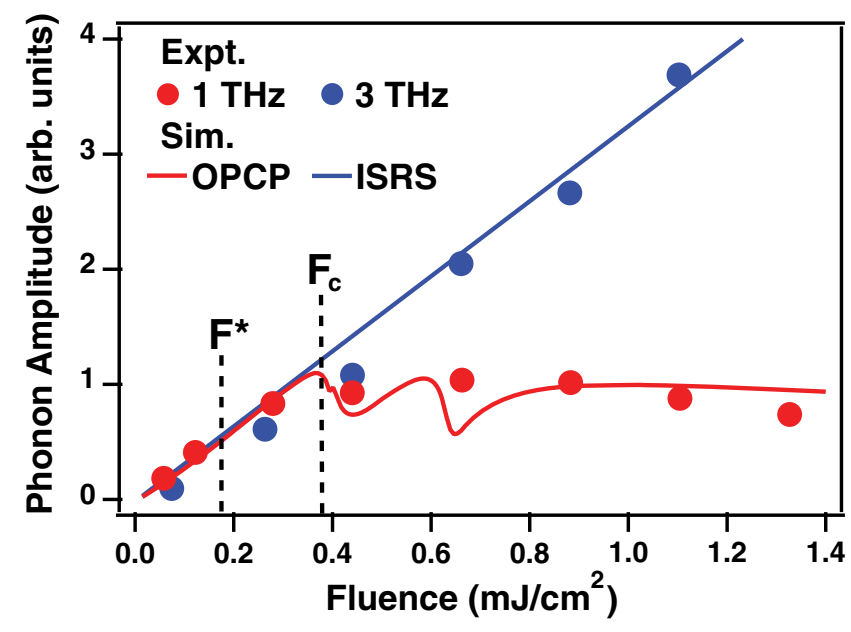

FIG. 3. Experimental pump fluence dependence of the $1 \mathrm{THz}$ (red circles) and $3 \mathrm{THz}$ (blue circles) coherent phonon amplitudes in $\mathrm{Ta}_{2} \mathrm{NiSe}_{5}$ reproduced from Ref. [28]. Simulation results for an OPCP (red line) and a conventional ISRS phonon (blue line) are overlaid and horizontally scaled $(\alpha \approx 700)$ to match the experimental data. Vertical dashed lines mark the calculated $F_{c}$ and $F^{*}$. The nonmonotonic behavior of the OPCP amplitude just above $F_{c}$ arises from strong feedback between $\Phi$ and $X$ immediately after excitation [35]. 
$\sim 0.4 \mathrm{~mJ} / \mathrm{cm}^{2}$, consistent with an order parameter reversal. By overlaying our simulation results [35] atop these experimental curves, we find close agreement (Fig. 3).

Our theory also predicts that the exciton condensate should be transiently quenched $[m(t) \rightarrow 0]$ above a critical fluence $F=F^{*}$ where the condition $f(t)=|m|$ is satisfied. Equations (2) and (3) do not constrain $F^{*}$ to coincide with $F_{c}$ and our simulation shows that $F^{*}$ is clearly lower than $F_{c}$ in $\mathrm{Ta}_{2} \mathrm{NiSe}_{5}$ (Fig. 3). Recently Tang et al. [30] performed time- and angle-resolved photoemission spectroscopy (TR-ARPES) measurements on $\mathrm{Ta}_{2} \mathrm{NiSe}_{5}$ at $T=30 \mathrm{~K}$ and tracked the dynamics of the charge gap, a measure of $\Phi$, immediately after pumping with $1.77 \mathrm{eV}$ light polarized perpendicular to the chain direction (equivalent geometry to Ref. [28]). They found that the instantaneous gap size decreases linearly with increasing pump fluence and saturates above $0.29 \mathrm{~mJ} / \mathrm{cm}^{2}$, which was interpreted as the point where $\Phi$ transiently collapses. The fact that this fluence is lower than $0.4 \mathrm{~mJ} / \mathrm{cm}^{2}$ and is expected to be even lower if the experiment were conducted at $120 \mathrm{~K}$ is consistent with our theory.

The dynamical nature of the order parameter reversal process suggests that it can be controlled not merely by the total pump energy deposited, but also by its distribution in time. To show this, we consider a situation where the sample is pumped by two identical pulses separated by time $\delta t$, with individual fluences $F<F_{c}$ but $2 F>F_{c}$. For $\delta t \rightarrow 0$ the system is effectively pumped by a single pulse exceeding $F_{c}$ and so reversal occurs, while for $\delta t \rightarrow \infty$, the system relaxes back to the initial ground state before the second pulse arrives and so no reversal occurs. To qualitatively understand the behavior at intermediate $\delta t$ values, where the system is still dynamically evolving when the second pulse arrives, we recall that in the single-pulse case, switching occurs once the OPCP amplitude is large enough to change the sign of $X$. Therefore, in the two-pulse case, switching possibly occurs if coherent oscillations of the OPCP induced by the first pulse can be sufficiently amplified by the second time-delayed pulse.

Previous studies have shown that impulsively and displacively excited conventional Raman-active phonons can be coherently amplified (suppressed) by a second pump pulse when $\delta t$ is an integer (half-integer) multiple of the phonon period [49-54], dubbed in-phase (IP) and out-of-phase (OP) pumping, respectively. Therefore, we simulated the effects of both IP and OP pumping on the order parameters of $\mathrm{Ta}_{2} \mathrm{NiSe}_{5}$ using the same materials parameters as before. We chose to simulate the OPCP at $\omega_{0} / 2 \pi=2 \mathrm{THz}$ rather than at $1 \mathrm{THz}$ because recent TRARPES data show that the most pronounced modulations of the valence band maximum occur at $2 \mathrm{THz}$, suggesting strong coupling to $\Phi[18]$. As shown in Fig. 4(a), pumping by a single pulse with $F$ slightly less than

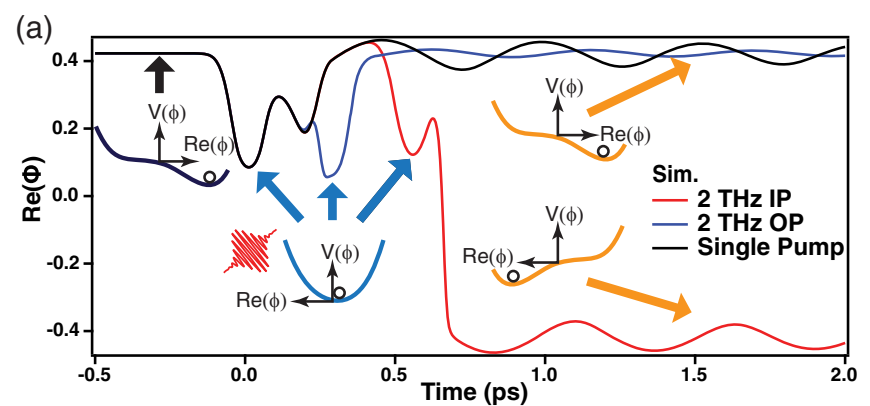

(b)

(c)
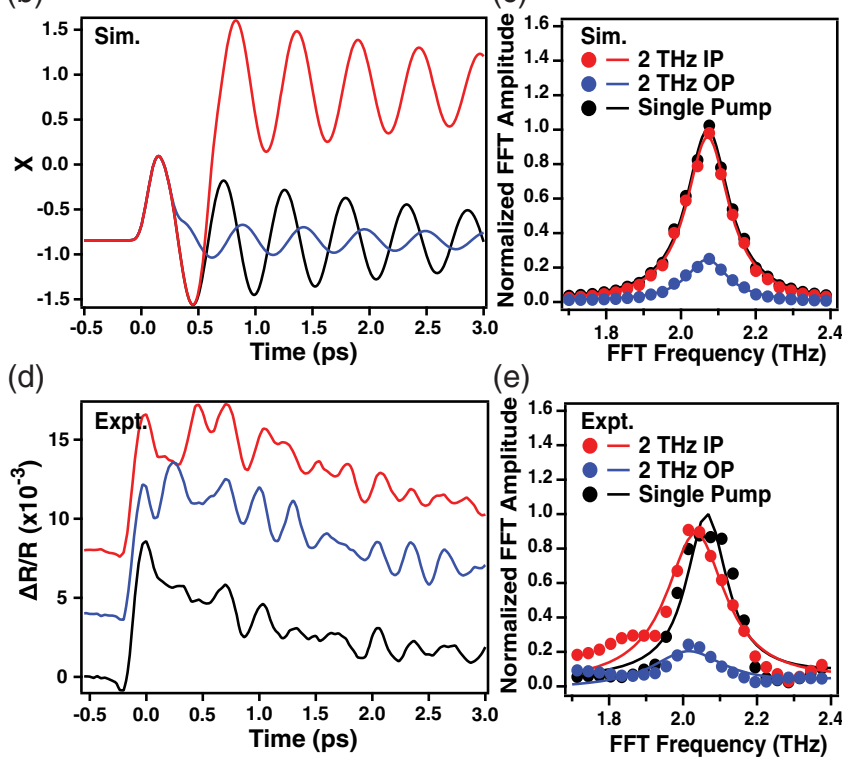

(e)

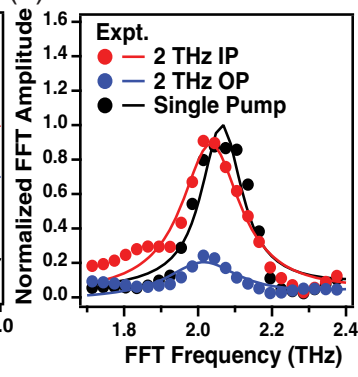

FIG. 4. Simulated time evolution of (a) $\operatorname{Re}(\Phi)$ and (b) $X$ following single-pulse pumping (black) and two-pulse OP (blue) and IP (red) pumping of the $2 \mathrm{THz}$ phonon using $F=0.96 F_{c}$ and the same microscopic parameters as in Figs. 2 and 3. The calculated instantaneous electronic potential is displayed at several select times. (c) Normalized FFT of the traces shown in (b). Each curve is normalized by the peak value of the singlepulse pumping curve. (d) Reflectivity transients measured from $\mathrm{Ta}_{2} \mathrm{NiSe}_{5}$ under the same pumping conditions used in the simulations. Curves are vertically offset for clarity. (e) Normalized FFT of the traces shown in (d).

$F_{c}$ causes a rapid but incomplete reduction of $\Phi$, followed by a slower recovery back to its original value on a timescale set by the damping of the $2 \mathrm{THz}$ phonon. For the two-pulse case, OP pumping of the 2 THz phonon similarly leads to partial suppression of $\Phi$ without reversal, but reversal is achieved with IP pumping. This phenomenon is again manifested through an unconventional behavior of the OPCP. As shown in Figs. 4(b) and 4(c), OP pumping leads to suppression of the $2 \mathrm{THz}$ phonon amplitude relative to the single pump case, resembling a conventional phonon because the oscillation is around the initial potential minimum. But, in contrast to conventional behavior, IP pumping does not lead to further amplification once $X$ is excited to the opposite minimum. 
To verify this prediction, we performed transient optical reflectivity measurements on $\mathrm{Ta}_{2} \mathrm{NiSe}_{5}$ single crystals [35] using two identical pump pulses ( $\sigma=80 \mathrm{fs}$ ) with variable $\delta t$. The light was polarized perpendicular to the chain direction and the fluence of each pulse was tuned slightly below $F_{c}$ to match our simulations. We chose a pump photon energy of $1 \mathrm{eV}$ to enhance the $2 \mathrm{THz}$ oscillations [35]. Figure 4(d) shows the fractional change in reflectivity $(\Delta R / R)$ versus time for both IP and OP pumping of the $2 \mathrm{THz}$ phonon, as well as for pumping with only a single pulse. All three curves exhibit fast $(\sim 1 \mathrm{ps})$ exponential decay following pump excitation, corresponding to the charge relaxation process. Oscillations from the beating of several coherently excited phonons are also clear. A FFT of the data shows the most pronounced peaks at 2, 3, and $3.8 \mathrm{THz}$ [35]. A focus on the $2 \mathrm{THz}$ mode reveals that OP pumping strongly suppresses its amplitude relative to the single-pump case, whereas IP pumping does not amplify it [Fig. 4(e)], in quantitative agreement with our simulations [Fig. 4(c)]. In contrast, strong amplification occurs for the 3 and 3.8 THz modes [35], consistent with their uncoupled nature.

Our field theory description of the EI order parameter goes beyond the phenomenological time-dependent Landau theory [55-58] in that it allows the order parameter to explore the tilted Mexican-hat potential in the complex plane and can be naturally linked to microscopic parameters of the underlying lattice model. While more details including extension beyond the mean-field limit $(\nabla \neq 0)$, temperature dependence with $T>0$, diffusion perpendicular to the surface [57], time-dependent damping [55], and anharmonic phonon coupling [59] can be added to refine the simulations, our minimal microscopic theory already captures the most salient physics and experimental features. These ideas and dynamical protocols apply not only to excitonic insulators, but also to any system featuring a continuous-symmetry-breaking electronic order parameter induced by coupling to a structural order parameter, such as a charge ordered system coupled to a Peierls distortion or an orbital ordered system coupled to a Jahn-Teller distortion. Therefore, the OPCP behavior revealed here may be a general fingerprint of electronic order parameter switching.

We thank Rick Averitt, Edoardo Baldini, Swati Chaudhary, Nuh Gedik, Xinwei Li, Tianwei Tang, and Alfred Zong for useful discussions. D. H. and J. G. C. acknowledge support from the DARPA DRINQS program (Grant No. D18AC00014). D. H. also acknowledges support for instrumentation from the Institute for Quantum Information and Matter, a NSF Physics Frontiers Center (PHY-1733907). M. B. acknowledges the support from the Department of Energy under Award No. DE-SC0019166. T. K. acknowledges the support by the Yamada Science Foundation Fellowship for Research Abroad and JSPS Overseas Research Fellowships.
* Corresponding author. dhsieh@caltech.edu

[1] C.-H. Lambert, S. Mangin, B. S. D. C. S. Varaprasad, Y. K. Takahashi, M. Hehn, M. Cinchetti, G. Malinowski, K. Hono, Y. Fainman, M. Aeschlimann, and E. E. Fullerton, Science 345, 1337 (2014).

[2] I. Tudosa, C. Stamm, A. B. Kashuba, F. King, H. C. Siegmann, J. Stöhr, G. Ju, B. Lu, and D. Weller, Nature (London) 428, 831 (2004).

[3] T. Gerrits, H. A. M. van den Berg, J. Hohlfeld, L. Bär, and T. Rasing, Nature (London) 418, 509 (2002).

[4] C. D. Stanciu, F. Hansteen, A. V. Kimel, A. Kirilyuk, A. Tsukamoto, A. Itoh, and T. Rasing, Phys. Rev. Lett. 99, 047601 (2007).

[5] K. Vahaplar, A. M. Kalashnikova, A. V. Kimel, D. Hinzke, U. Nowak, R. Chantrell, A. Tsukamoto, A. Itoh, A. Kirilyuk, and T. Rasing, Phys. Rev. Lett. 103, 117201 (2009).

[6] I. Radu, K. Vahaplar, C. Stamm, T. Kachel, N. Pontius, H. A. Dürr, T. A. Ostler, J. Barker, R. F. L. Evans, R. W. Chantrell, A. Tsukamoto, A. Itoh, A. Kirilyuk, T. Rasing, and A. V. Kimel, Nature (London) 472, 205 (2011).

[7] A. V. Kimel, B. A. Ivanov, R. V. Pisarev, P. A. Usachev, A. Kirilyuk, and T. Rasing, Nat. Phys. 5, 727 (2009).

[8] S. Manz, M. Matsubara, T. Lottermoser, J. Büchi, A. Iyama, T. Kimura, D. Meier, and M. Fiebig, Nat. Photonics 10, 653 (2016).

[9] S. Schlauderer, C. Lange, S. Baierl, T. Ebnet, C. P. Schmid, D. C. Valovcin, A. K. Zvezdin, A. V. Kimel, R. V. Mikhaylovskiy, and R. Huber, Nature (London) 569, 383 (2019).

[10] S. Fahy and R. Merlin, Phys. Rev. Lett. 73, 1122 (1994).

[11] T. Qi, Y.-H. Shin, K.-L. Yeh, K. A. Nelson, and A. M. Rappe, Phys. Rev. Lett. 102, 247603 (2009).

[12] R. Mankowsky, A. von Hoegen, M. Först, and A. Cavalleri, Phys. Rev. Lett. 118, 197601 (2017).

[13] D. Jérome, T. M. Rice, and W. Kohn, Phys. Rev. 158, 462 (1967).

[14] C. Lian, S.-J. Zhang, S.-Q. Hu, M.-X. Guan, and S. Meng, Nat. Commun. 11, 43 (2020).

[15] T. I. Larkin, A. N. Yaresko, D. Pröpper, K. A. Kikoin, Y. F. Lu, T. Takayama, Y.-L. Mathis, A. W. Rost, H. Takagi, B. Keimer, and A. V. Boris, Phys. Rev. B 95, 195144 (2017).

[16] T. Kaneko, T. Toriyama, T. Konishi, and Y. Ohta, Phys. Rev. B 87, 035121 (2013).

[17] A. Nakano, T. Hasegawa, S. Tamura, N. Katayama, S. Tsutsui, and H. Sawa, Phys. Rev. B 98, 045139 (2018).

[18] T. Suzuki, Y. Shinohara, Y. Lu, M. Watanabe, J. Xu, K. L. Ishikawa, H. Takagi, M. Nohara, N. Katayama, H. Sawa, M. Fujisawa, T. Kanai, J. Itatani, T. Mizokawa, S. Shin, and K. Okazaki, arXiv:2002.10037.

[19] M. Fiebig, T. Lottermoser, D. Fröhlich, and S. Kallenbach, Opt. Lett. 29, 41 (2004).

[20] M. Fiebig, D. Fröhlich, G. Sluyterman v. L., and R. V. Pisarev, Appl. Phys. Lett. 66, 2906 (1995).

[21] M. Fiebig, D. Fröhlich, B. B. Krichevtsov, and R. V. Pisarev, Phys. Rev. Lett. 73, 2127 (1994).

[22] M. Fiebig, T. Lottermoser, D. Fröhlich, A. V. Goltsev, and R. V. Pisarev, Nature (London) 419, 818 (2002).

[23] B. B. Van Aken, J.-P. Rivera, H. Schmid, and M. Fiebig, Nature (London) 449, 702 (2007). 
[24] M. Matsubara, S. Manz, M. Mochizuki, T. Kubacka, A. Iyama, N. Aliouane, T. Kimura, S. L. Johnson, D. Meier, and M. Fiebig, Science 348, 1112 (2015).

[25] A. Kogar, M. S. Rak, S. Vig, A. A. Husain, F. Flicker, Y. I. Joe, L. Venema, G. J. MacDougall, T. C. Chiang, E. Fradkin, J. van Wezel, and P. Abbamonte, Science 358, 1314 (2017).

[26] Y. Wakisaka, T. Sudayama, K. Takubo, T. Mizokawa, M. Arita, H. Namatame, M. Taniguchi, N. Katayama, M. Nohara, and H. Takagi, Phys. Rev. Lett. 103, 026402 (2009).

[27] K. Seki, Y. Wakisaka, T. Kaneko, T. Toriyama, T. Konishi, T. Sudayama, N. L. Saini, M. Arita, H. Namatame, M. Taniguchi, N. Katayama, M. Nohara, H. Takagi, T. Mizokawa, and Y. Ohta, Phys. Rev. B 90, 155116 (2014).

[28] D. Werdehausen, T. Takayama, M. Höppner, G. Albrecht, A. W. Rost, Y. Lu, D. Manske, H. Takagi, and S. Kaiser, Sci. Adv. 4, eaap8652 (2018).

[29] S. Mor, M. Herzog, J. Noack, N. Katayama, M. Nohara, H. Takagi, A. Trunschke, T. Mizokawa, C. Monney, and J. Stähler, Phys. Rev. B 97, 115154 (2018).

[30] T. Tang, H. Wang, S. Duan, Y. Yang, C. Huang, Y. Guo, D. Qian, and W. Zhang, Phys. Rev. B 101, 235148 (2020).

[31] B. Zenker, H. Fehske, and H. Beck, Phys. Rev. B 90, 195118 (2014).

[32] Y. Tanaka, M. Daira, and K. Yonemitsu, Phys. Rev. B 97, 115105 (2018).

[33] Y. Murakami, D. Golež, M. Eckstein, and P. Werner, Phys. Rev. Lett. 119, 247601 (2017).

[34] T. Tanabe, K. Sugimoto, and Y. Ohta, Phys. Rev. B 98, 235127 (2018).

[35] See Supplemental Material at http://link.aps.org/ supplemental/10.1103/PhysRevLett.125.267602 for extensive derivation of theory and simulation and experimental details, which contains Refs. [36-45].

[36] A. Kamenev, Field Theory of Non-Equilibrium Systems (Cambridge University Press, Cambridge, England, 2011).

[37] S. Mor, M. Herzog, D. Golež, P. Werner, M. Eckstein, N. Katayama, M. Nohara, H. Takagi, T. Mizokawa, C. Monney, and J. Stähler, Phys. Rev. Lett. 119, 086401 (2017).

[38] K. Okazaki, Y. Ogawa, T. Suzuki, T. Yamamoto, T. Someya, S. Michimae, M. Watanabe, Y. Lu, M. Nohara, H. Takagi, N. Katayama, H. Sawa, M. Fujisawa, T. Kanai, N. Ishii, J. Itatani, T. Mizokawa, and S. Shin, Nat. Commun. 9, 4322 (2018).

[39] M. D. Watson, I. Marković, E. A. Morales, P. L. Fèvre, M. Merz, A. A. Haghighirad, and P. D. C. King, Phys. Rev. Research 2, 013236 (2020).
[40] Y. F. Lu, H. Kono, T. I. Larkin, A. W. Rost, T. Takayama, A. V. Boris, B. Keimer, and H. Takagi, Nat. Commun. 8, 14408 (2017).

[41] H. J. Zeiger, J. Vidal, T. K. Cheng, E. P. Ippen, G. Dresselhaus, and M.S. Dresselhaus, Phys. Rev. B 45, 768 (1992).

[42] A. V. Kuznetsov and C. J. Stanton, Phys. Rev. Lett. 73, 3243 (1994).

[43] O. V. Misochko and M. V. Lebedev, Phys. Rev. B 94, 184307 (2016).

[44] S. Hunsche, K. Wienecke, T. Dekorsy, and H. Kurz, Phys. Rev. Lett. 75, 1815 (1995).

[45] P. Tangney and S. Fahy, Phys. Rev. Lett. 82, 4340 (1999).

[46] Y. Murakami, D. Golež, T. Kaneko, A. Koga, A. J. Millis, and P. Werner, Phys. Rev. B 101, 195118 (2020).

[47] T. E. Stevens, J. Kuhl, and R. Merlin, Phys. Rev. B 65, 144304 (2002).

[48] A. Melnikov, O. Misochko, and S. Chekalin, Phys. Lett. A 375, 2017 (2011).

[49] M. Hase, K. Mizoguchi, H. Harima, S. Nakashima, M. Tani, K. Sakai, and M. Hangyo, Appl. Phys. Lett. 69, 2474 (1996).

[50] M. Hase, T. Itano, K. Mizoguchi, and S. ichi Nakashima, Jpn. J. Appl. Phys. 37, L281 (1998).

[51] E. D. Murray, D. M. Fritz, J. K. Wahlstrand, S. Fahy, and D. A. Reis, Phys. Rev. B 72, 060301(R) (2005).

[52] O. V. Misochko, M. V. Lebedev, H. Schäfer, and T. Dekorsy, J. Phys. Condens. Matter 19, 406220 (2007).

[53] A. Q. Wu and X. Xu, Appl. Phys. Lett. 90, 251111 (2007).

[54] Y.-H. Cheng, F. Y. Gao, S. W. Teitelbaum, and K. A. Nelson, Phys. Rev. B 96, 134302 (2017).

[55] T. Huber, S. O. Mariager, A. Ferrer, H. Schäfer, J. A. Johnson, S. Grübel, A. Lübcke, L. Huber, T. Kubacka, C. Dornes, C. Laulhe, S. Ravy, G. Ingold, P. Beaud, J. Demsar, and S. L. Johnson, Phys. Rev. Lett. 113, 026401 (2014).

[56] A. Zong et al., Phys. Rev. Lett. 123, 097601 (2019).

[57] R. Yusupov, T. Mertelj, V. V. Kabanov, S. Brazovskii, P. Kusar, J.-H. Chu, I. R. Fisher, and D. Mihailovic, Nat. Phys. 6, 681 (2010).

[58] J. W. Harter, D. M. Kennes, H. Chu, A. de la Torre, Z. Y. Zhao, J.-Q. Yan, D. G. Mandrus, A. J. Millis, and D. Hsieh, Phys. Rev. Lett. 120, 047601 (2018).

[59] M. Hase, M. Kitajima, S.-i. Nakashima, and K. Mizoguchi, Phys. Rev. Lett. 88, 067401 (2002). 